\title{
Appendix 4: Script
}

Welcome, I am X, a ... here at XXX and I will be assisted today by .... to do a card game today. Before we go to the instructions I ask you to turn off any mobile phones and other mobile devices that can disturb the experiment. If you have a question, raise your hand, and I will address your question.

The duration of the experiment is a maximum of 2 hours. You earned a 10 dollar Amazon gift card by showing up here on time. Dependent on the decisions you make you can earn additional goods as explained in the instructions.

The game we are gonna play is called Port of Mars. You are each a member of Generation Zero in the first long-term human community of Mars. Your goal is to keep the community alive while earning individual points by achieving Accomplishments that makes Mars history.

Each of you has a special role in Generation Zero. We have five roles, namely Curator, Pioneer, Researcher, Politician, and Entrepreneur. Those roles invest time to get influence cards for Culture, Legacy, Science, Government, and Finance, which are used to earn points.

The basic gest of the game is the following. Each round you have time to spend, say 10 hours a day, and you have to choose how much to invest in the community and how much in your personal interests. The upkeep level of the community needs to stay positive otherwise it is game over for all of you. You play an unknown number of rounds, and if you keep the community alive, you all get a special Port of Mars magnet. The person will get the most points during the game will also get a special Port of Mars water bottle, and of course a place in the Port of Mars Hall of fame.

You have instructions in front of you and I will ask you to read them in a minute. But first I want to make sure you know what are the time blocks (show them) that you invest in activities to earn influence cards. With the right set of influence cards you can earn accomplishment cards, and you will have each round 3 accomplishment cards in front of you.

When you choose to invest your time blocks you have 6 options as you see on your play sheet in front of you. In the left top corner, you place the number of time blocks you want to invest in upkeep. The rest is to obtain influence cards. Each player has one influence topic they are good at earning (costs 2 time-blocks to earn 1 influence card), two influence topics they can earn (costs 3 time-blocks to earn 1 influence card), and two influence topics they can't earn at all. You see this at the right part of your play sheet. To get influence cards for those topics, you will have to trade with other players. 
One special card that needs explanation is an accomplishment card with Upkeep symbol. [how example] The cost, in that case, is a reduction of upkeep for the group, not that you use upkeep time investment to collect the points.

So read the instructions carefully, and then we can address some of the questions you have.

[participants read instructions]

Are there any questions?

\section{[Address questions]}

We know that not everything might be clear at the start of round one. That is also the reason, we do not use an event card in round 1. So let's start round 1 with an upkeep index of 100. The first thing we do is to reduce it with 25 units, leading to 75 units left.

Now reveal 3 accomplishment cards by taking them from your stack.

You all have 10 time-blocks to invest.

Now you have 5 minutes to discuss with the other players on what you plan to do which include potential trade arrangements. You can discuss whatever you want as long as you don't use any offensive language or make promises about side payments after the game.

Now you have to finalize your time allocation.

The observer will give the influence cards, and the data recorder will record your time investments.

Now you have time to trade influence cards.

Finally, you can spend your influence to earn points to buy accomplishment cards. You may also discard accomplishment cards now and the next round you can fill it up to 3 accomplishment cards.

.. [play the rest of the game]

This was the last round, but not the end of the experiment. You are asked to fill in carefully a brief survey and do brief interviews. When some of you get a brief interview the others will fill out the survey. 